\title{
Characterisation of osteophytes as an autologous bone graft source
}

\author{
AN EXPERIMENTAL STUDY IN VIVO AND IN VITRO
}

\section{K. Ishihara, \\ K. Okazaki, \\ T. Akiyama, \\ Y. Akasaki, \\ Y. Nakashima}

Graduate School

of Medical Science,

Kyushu University,

Fukuoka, Japan
K. Ishihara, MD, Postgraduate

Student,

K. Okazaki, MD, PhD, Associate

Professor,

- Y. Akasaki, MD, PhD, Asssistant

Professor,

- Y. Nakashima, MD, PhD,

Professor and Chairman,

Department of Orthopedic

Surgery, Graduate School

of Medical Science, Kyushu

University, Fukuoka, Japan.

- T. Akiyama, MD, Director,

Akiyama Clinic, Fukuoka, Japan.

Correspondence should be sent to K. Okazaki; email: okazaki@med. kyushu-u.ac.jp

doi: $10.1302 / 2046-3758.62 . B J R-$ 2016-0199.R1

Received: 8 August 2016;

Accepted: 28 November 2016

Bone Joint Res 2017;6:73-81.

\section{Objectives}

Osteophytes are products of active endochondral and intramembranous ossification, and therefore could theoretically provide significant efficacy as bone grafts. In this study, we compared the bone mineralisation effectiveness of osteophytes and cancellous bone, including their effects on secretion of growth factors and anabolic effects on osteoblasts.

\section{Methods}

Osteophytes and cancellous bone obtained from human patients were transplanted onto the calvaria of severe combined immunodeficient mice, with Calcein administered intraperitoneally for fluorescent labelling of bone mineralisation. Conditioned media were prepared using osteophytes and cancellous bone, and growth factor concentration and effects of each graft on proliferation, differentiation and migration of osteoblastic cells were assessed using enzyme-linked immunosorbent assays, MTS ((3-(4,5-dimethylthiazol-2-yl)5-(3-carboxymethoxyphenyl)-2-(4-sulfophenyl)-2H-tetrazolium)) assays, quantitative realtime polymerase chain reaction, and migration assays.

\section{Results}

After six weeks, the area of mineralisation was significantly higher for the transplanted osteophytes than for the cancellous bone $\left(43803 \mu \mathrm{m}_{2}\right.$, SD 14660 versus $9421 \mu \mathrm{m}_{2}$, SD 5032, $p=0.0184$, one-way analysis of variance). Compared with cancellous bone, the conditioned medium prepared using osteophytes contained a significantly higher amounts of transforming growth factor (TGF)- $\beta 1$ (471 pg/ml versus $333 \mathrm{pg} / \mathrm{ml}, \mathrm{p}=0.0001$, Wilcoxon rank sum test), bone morphogenetic protein (BMP)-2 $(47.75 \mathrm{pg} / \mathrm{ml}$ versus $32 \mathrm{pg} / \mathrm{ml}, \mathrm{p}=0.0214$, Wilcoxon rank sum test) and insulin-like growth factor (IGF)-1 $(314.5 \mathrm{pg} / \mathrm{ml}$ versus $191 \mathrm{pg} / \mathrm{ml}$, $p=0.0418$, Wilcoxon rank sum test). The stronger effects of osteophytes towards osteoblasts in terms of a higher proliferation rate, upregulation of gene expression of differentiation markers such as alpha- 1 type- 1 collagen and alkaline phosphate, and higher migration, compared with cancellous bone, was confirmed.

\section{Conclusion}

We provide evidence of favourable features of osteophytes for bone mineralisation through a direct effect on osteoblasts. The acceleration in metabolic activity of the osteophyte provides justification for future studies evaluating the clinical use of osteophytes as autologous bone grafts.

Cite this article: Bone Joint Res 2017;6:73-81.

Keywords: Osteophyte, Bone graft, Growth factor, Osteotomy

\section{Article focus}

- To prove our hypothesis in a biological sense, that osteophytes have greater mineralisation efficacy than cancellous bone using mice in vivo and osteoblast-like cells in vitro.
Key messages

- Six weeks after transplantation, osteophytes produced a greater amount of newly mineralised area around bone grafts, in vivo, than cancellous bone. 
- Osteophytes release more growth factors (transforming growth factor (TGF)- $\beta 1$, bone morphogenetic protein (BMP)-2, and insulin-like growth factor (IGF)1) than cancellous bone.

- Conditioned medium prepared from osteophytes stimulated greater proliferation, differentiation and migration of osteoblast-like cells in vitro, than medium prepared from cancellous bone.

\section{Strengths and limitations}

- To our knowledge, this is the first study to demonstrate the specific character of osteophytes as an autologous bone graft source.

- The principal limitation of our study is the small number of mice used in quantitative in vivo experiments, and the use of MG-63 and Saos-2 as osteoblast-like cells, in vitro, rather than primary osteoblasts isolated from humans.

\section{Introduction}

Bone defects requiring treatment are frequently encountered during various orthopaedic surgeries, including fracture reduction and fixation, arthroplasty and osteotomy. Bone grafts, such as autograft, allograft and artificial substitute materials, are options to fill these bone defects. Among these options, autografts provide a rich source of progenitor cells, bone morphogenetic proteins (BMPs) and growth factors which facilitate new bone formation. ${ }^{1,2}$ Autografts are therefore commonly used due to their osteogenic, osteoinductive and osteoconductive properties. The iliac crest is the most common source of bone autograft, providing a relatively easy harvest of a fair quantity of bone. However, harvesting in general increases surgical time and blood loss, as well as the overall cost of the procedure, and carries the risk for donor site morbidity which, at the iliac crest, includes infection, pain, haemorrhage, fracture, muscle weakness and nerve injury. ${ }^{3}$

Osteophytes are osteocartilaginous metaplastic tissues that generally form at the margins of osteoarthritic joints. 4,5 The development of osteophytes follows a basic pattern of endochondral and intramembranous bone formation, ${ }^{6,7}$ in which growth factors and BMPs play an important role. ${ }^{8-10}$ Considering the contribution of endochondral and intramembranous bone formation to osteophyte development, we hypothesised that osteophytes could provide sufficient osteogenic efficacy to be used as autologous bone grafts. From a clinical perspective, we conceived that a medial open-wedge high tibial osteotomy (OW-HTO), for the treatment of varus knee osteoarthritis (OA), would be ideal for the use of osteophyte bone grafting.

The OW-HTO procedure is well accepted in practice, with documented good clinical results since 1987.11 However, one of the concerns of OW-HTO procedures involves the residual gap at the osteotomy site, which can lead to several complications such as delayed bone union, loss of correction and breakage of the fixation plate and screws. ${ }^{12}$ Therefore, methods and materials that could accelerate bone union would be desirable. As a clinical study of feasibility, we have been using osteophytes as an autologous bone graft to fill the gap at the osteotomy site during OW-HTO procedures, harvesting the osteophytes arthroscopically from the intercondylar notch or medial edge of the femur, and grafting them around the lateral hinge area of the osteotomy site. Among 11 consecutive cases of implantation of osteophytes at the osteotomy site, bone healing was achieved, at a mean 5.3 weeks post-operatively,,$^{13}$ an accelerated rate of bone union than previously reported. ${ }^{12}$ Our clinical experience to date supports our hypothesis regarding the favourable bone-forming efficacy of osteophytes, and of their potential usefulness as bone grafts. Therefore, the aim of our study was to compare the osteogenic efficacy of osteophytes and cancellous bone grafts, specifically addressing the following three questions: do osteophytes produce more bone mineralisation than cancellous bone in vivo?; do osteophytes secrete more growth factors than cancellous bone?; and do factors released from osteophytes have stronger effects on proliferation, differentiation and chemotaxis of osteoblasts than those from cancellous bone in vitro?

\section{Materials and Methods}

Mice and human samples. All animal experiments followed protocols approved by the institutional animal care and use committee of our institution (approval number: A25-216-0). A total of 18 eight-week-old male CB17-severe combined immunodeficient (SCID) mice (Charles River Laboratories Japan Inc, Yokohama, Japan) were used for this study. Animals were housed in separate cages in the animal centre of our institution, with free access to water and standard food provided.

Both human osteophytes and cancellous bone tissue were obtained from 19 female patients during total knee arthroplasty for OA. In order for the surgical samples to be used in experiments, written consent was obtained from every patient. Nine of the osteophyte samples were used for in vivo transplantation and ten were used for incubation in a conditioned medium. Although it is relatively easy to distinguish osteophytes from normal bone at the femoral condyle, tibial osteophytes blend into the tibial plateau, such that the transition between normal bone and osteophyte cannot be determined. ${ }^{14}$ Therefore, we exclusively used femoral osteophytes in our study. Cancellous bone was harvested from the mid-portion of bone resected from the chamfer cut on the femoral condyle. These procedures were approved by the institutional review board for clinical research of our institution (approval number: 25-173). 
In vivo transplantation. Using an aseptic technique, human osteophytes and cancellous bone were divided into $0.05 \mathrm{~g}$ pieces and transplanted onto the calvaria of mice under general anaesthesia. An incision was made on the head of CB17-SCID mice, the periosteum peeled off, and the osteophyte or cancellous bone was transplanted into nine randomised mice for each graft type. The wound was closed with a surgical suture.

Histological analysis. Three mice in each of the two experimental groups, osteophyte and cancellous bone, were killed at three and six weeks following transplantation, for histological analysis. The whole calvaria were fixed in $4 \%$ paraformaldehyde, decalcified with $0.5 \mathrm{M}$ ethylenediaminetetraacetic acid and embedded in paraffin wax. Coronal sections ( $5 \mu \mathrm{m}$ thick) were stained with Safranin O, fast green and Weigert's iron haematoxylin, and examined by light microscopy. Concurrently, consecutive slices were stained with haematoxylin and eosin (H\&E).

Micro-CT scanning. For morphological observation, specimens were scanned by micro CT (Hitachi Aloka Medical, Tokyo, Japan) at both time points of histological analysis. Mineralisation around the graft. The remaining three mice in each group were killed for histomorphometrical analysis at six weeks following transplantation. Calcein (Dojindo Lab, Kumamoto, Japan) was subcutaneously administered at a dose of $10 \mathrm{mg} / \mathrm{kg}$ of body weight at five weeks post-transplantation for fluorescent labelling of the area of mineralisation. For analysis, the calvaria was fixed in $70 \%$ ethanol, treated with Villanueva bone stain for six days, dehydrated in graded concentrations of ethanol, and embedded in methyl-methacrylate (Wako Pure Chemical Industries, Osaka, Japan) without bone decalcification. The area of fluorescence and total area of the graft were measured by fluorescence microscope with image processing software (BZ-X700, Keyence Co, Osaka, Japan) and the ratio of labelled area:total area of the graft, was calculated.

Osteophyte- and cancellous bone-conditioned medium. Osteophytes and cancellous bone were milled and placed into sterile dishes containing Dulbecco's Modified Eagle Medium (DMEM; Life Technologies, Grand Island, New York) supplemented with $100 \mathrm{U} / \mathrm{mL}$ penicillin and 100 $\mu \mathrm{g} / \mathrm{mL}$ streptomycin (Sigma-Aldrich, St. Louis, Missouri), but with no serum. A ratio of $3 \mathrm{~g}$ of osteophytes or cancellous bone (wet weight) per $10 \mathrm{ml}$ of culture medium was used. After a 24-hour incubation period, the osteophyteconditioned medium and cancellous bone-conditioned medium were collected.

Enzyme-linked immunosorbent assay. The amount of transforming growth factor (TGF)- $\beta 1$, BMP-2 and insulinlike growth factor (IGF)-1 in the osteophyte-conditioned medium and cancellous bone-conditioned medium were measured using Quantikine Colorimetric Sandwich enzyme-linked immunosorbent assay (ELISA) kits (R\&D Systems, Minneapolis, Minnesota) in accordance with the manufacturer's protocol. To activate latent TGF- $\beta 1$ for measurement, the conditioned medium was heated to $85^{\circ} \mathrm{C}$ for ten minutes. Data from ten independent samples were analysed.

Cell culture, RNA extraction and real-time reverse transcriptase polymerase chain reaction. The human osteosarcoma cell lines, MG-63 and Saos-2, were purchased from ATCC (Manassas, Virginia) and maintained in DMEM supplemented with $10 \%$ foetal bovine serum (FBS). For gene expression analysis, cell lines $\left(5 \times 10^{4} /\right.$ well, 24 -well plate) were exposed to the osteophyte-conditioned medium or cancellous bone-conditioned medium for 72 hours. The osteophyte-conditioned medium and cancellous bone-conditioned medium were diluted three-fold, using DMEM with $10 \%$ FBS. As an untreated control, the cells were exposed to DMEM with 6.7\% FBS. Total RNA was isolated from cultured cells using the RNeasy Mini Kit (Qiagen, Hilden, Germany), and was reverse-transcripted using the PrimeScript Reverse Transcriptase (RT) reagent kit (Takara Bio, Shiga, Japan) to make single-stranded cDNA. Quantitative real-time RT-polymerase chain reaction (PCR) was performed with the LightCycler 2.0 System (F. Hoffmann-La Roche AG, Basel, Switzerland) using SYBR Premix Ex Taq II (Takara Bio Inc., Shiga, Japan). The primer sequences are included in the supplementary material. For analysis, data were corrected for expression of the housekeeping gene GAPDH and the results are shown in the figures. Data from ten independent samples were analysed.

Proliferation assay. To evaluate the rate of cell proliferation, MG-63 cells ( $1.5 \times 10^{3} /$ well, 96 -well plate) were incubated in the osteophyte-conditioned medium or cancellous bone-conditioned medium, which were diluted three-fold, using DMEM without FBS or DMEM without FBS as an untreated control, for up to 36 hours. Viable cells were identified using CellTiter 96 AQueous One Solution Cell Proliferation Assay (Promega, Madison, Wisconsin) according to the manufacturer's protocol. Data were corrected for the absorbance at time zero. Data from ten independent samples were analysed.

Migration assay. A migration assay was performed using Transwell chambers (Corning Costar Corp., Cambridge, Massachusetts), with $6.5 \mathrm{~mm}$-diameter polycarbonate filters ( $8 \mu \mathrm{m}$ pore size). Polyvinylpyrrolidone-free polycarbonate filters in the upper chamber were coated with type 1 collagen (Nitta Gelatin, Inc., Osaka, Japan) and used as a cell permeable membrane. MG-63 cells $(1.0 \times$ $105 /$ well) were suspended in $200 \mu \mathrm{L}$ serum-free DMEM and seeded in the upper chamber. The lower chamber was filled with $600 \mu \mathrm{L}$ of osteophyte-conditioned medium or cancellous bone-conditioned medium, which were diluted three-fold using DMEM without FBS or DMEM without FBS as an untreated control. MG-63 cells were allowed to migrate for six hours at $37^{\circ} \mathrm{C}$ in $5 \% \mathrm{CO}_{2}$. Cells which migrated to the lower side of the filter were 


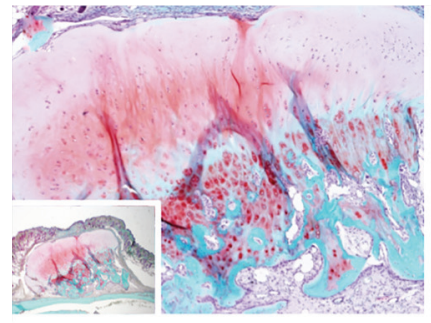

Fig. 1a

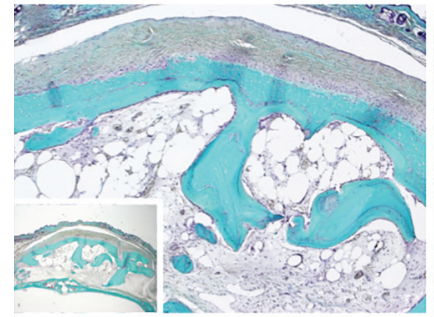

Fig. $1 \mathrm{~b}$

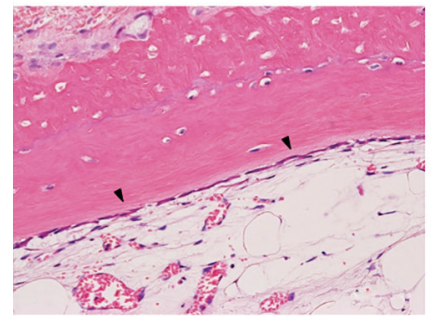

Fig. 1c

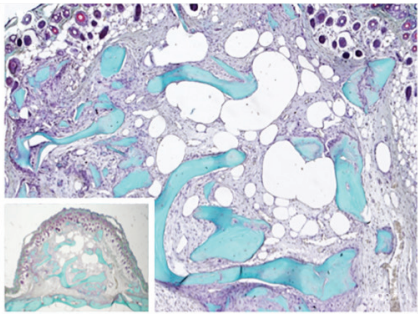

Fig. $1 \mathrm{~d}$

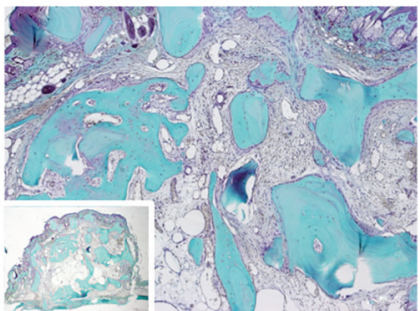

Fig. 1e

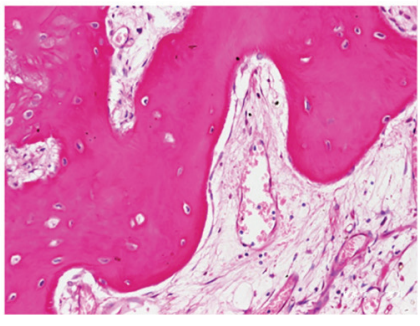

Fig. $1 \mathrm{f}$
Safranin O-fast green staining shown at (a) three weeks and (b) six weeks after osteophyte transplantation and, similarly, at (d) three weeks and (e) six weeks after cancellous bone transplantation (original magnification, $\times 80$ ). (c) Haematoxylin and eosin (H\&E) staining, six weeks after osteophyte transplantation, is shown, with the arrowheads indicating bone lining cells at the edge of the bony structure of the osteophyte (original magnification, $\times 400$ ). (f) H\&E staining, six weeks after cancellous bone transplantation, is shown (original magnification, $\times 400$ ).

fixed with methanol and stained using the Diff-Quik kit (Sysmex Co., Kobe, Japan). The cells that migrated to the lower surface of the filter were counted in three randomly selected microscopic fields $(\times 400)$, with the average of three measurements used for analysis. Data from ten independent samples were analysed.

Statistical analysis. All parameters were tested for normality with the Shapiro-Wilk test, with normally distributed variables first assessed using Levene's test. If appropriate, datasets with statistically significant differences from control values were analysed using a oneway analysis of variance (ANOVA), followed by Tukey's post hoc test for multiple comparisons. The results are reported as mean and standard deviation (SD). Wilcoxon rank sum test was used to analyse specific sample pairs for significant differences, such as the ELISA for each conditioned medium. The results are reported as median and interquartile range. Statistical analyses were conducted using JMP (version 11.0; SAS Institute Inc, Cary, North Carolina). A p-value $<0.05$ was considered statistically significant.

\section{Results}

Histological changes of transplanted osteophytes and cancellous bone in vivo. At three weeks following transplantation, histological assessment confirmed staining of the extracellular matrix of the cartilaginous structure of the transplanted osteophyte by Safranin O (Fig. 1a). After six weeks, the staining with Safranin O disappeared and was replaced by tissue stained with fast green (Fig. 1b), with bone lining cells visible at the edge of the bony structure in a high-power light microscope field (Fig. 1c). In contrast, no obvious morphological changes were observed by Safranin $O$ staining of the cancellous bone at three and six weeks post-transplantation (Figs $1 \mathrm{~d}$ and $1 \mathrm{e}$ ), with relatively sparse bone lining cells visible (Fig. 1f). Figures shown are representative of the full dataset.

Mineralisation potential of osteophyte and cancellous bone. Representative images of the Villanueva bone staining, obtained by Calcein labelling six weeks posttransplantation, are shown in Figure 2. Under fluorescence microscopy, the green line, indicative of mineralisation that is due to new bone deposition, was visible over almost the entire circumference of the transplanted osteophyte (Fig. 2b), while being visible in only a limited area of the cancellous bone transplantation (Fig. 2f). Much of the mineralisation is also observed on the surface of the calvarial bone in both groups. As it was observed not only below the graft, but also over the entire circumference of the calvarial bone, we suggest that it likely represents normal local intramembranous ossification. On micro-CT performed at three (Fig. 2c) and six (Fig. 2d) weeks posttransplantation, newly formed bone was confirmed only for the osteophyte transplantation at six weeks.

On quantitative analysis, the area of fluorescence in the grafted mass was significantly larger in the osteophyte group (43803 $\mu_{2}$, SD 14660 versus $9421 \mu \mathrm{m}_{2}$, SD 5032, $p=0.0184$; Fig. 3). The ratio of the labelled area to the total area of the graft was also significantly higher in the osteophyte group than in the cancellous bone group $(0.788 \%$, SD $0.101 \%$ versus $0.127 \%$, SD $0.03 \%, p=0.0035$ ). Therefore, osteophyte grafts continued to promote active bone formation even at five weeks post-transplantation, which is when the Calcein was administered.

\section{Growth factor secretion and anabolic effects on osteoblasts} in vitro. Quantitative analysis by ELISA of the osteophyteconditioned medium and cancellous bone-conditioned medium revealed a higher total amount of growth factors such as TGF- $\beta 1$, BMP- 2 , and IGF- 1 for the osteophyte than cancellous bone preparations (Fig. 4).

MTS assays of the cultured MG-63 cells in the osteophyte-conditioned medium, cancellous boneconditioned medium and untreated control medium confirmed a higher rate of proliferation in the osteophyteconditioned medium, compared with the untreated control medium throughout the period up to 36 hours (Fig. 5). After a 36-hour incubation period, the proliferation rate 


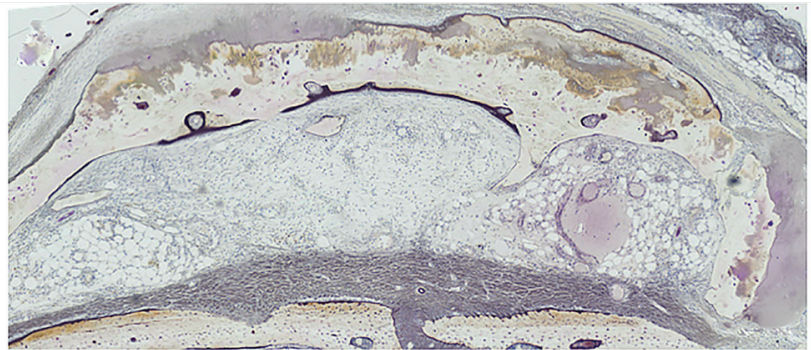

Fig. $2 a$

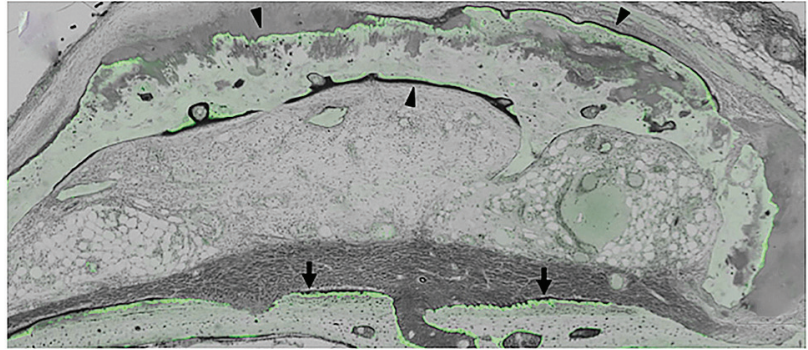

Fig. $2 b$

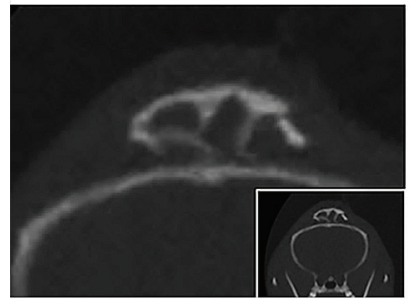

Fig. 2c

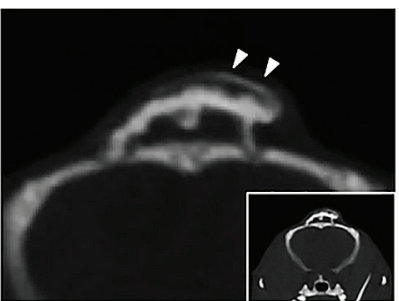

Fig. $2 d$

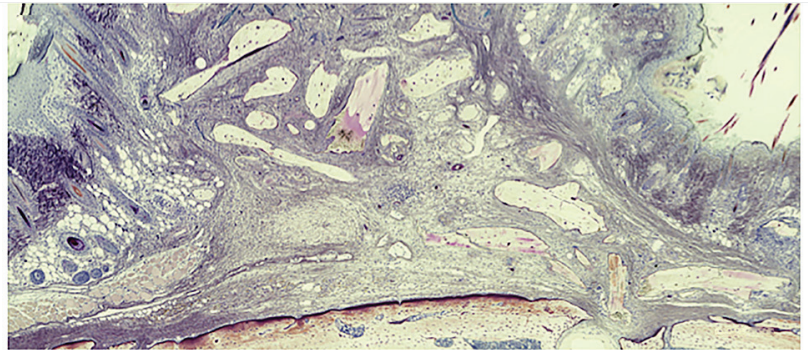

Fig. $2 \mathrm{e}$

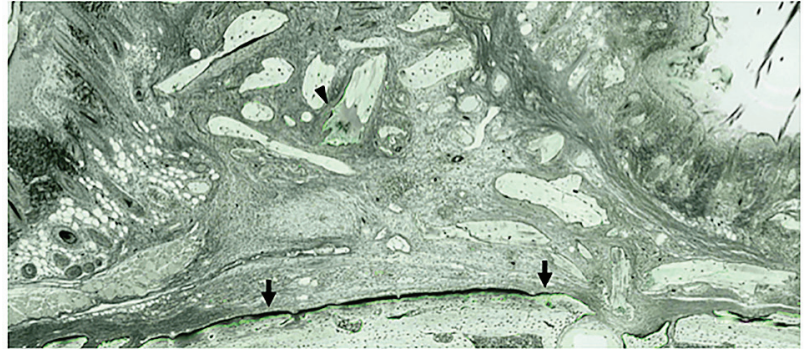

Fig. $2 f$

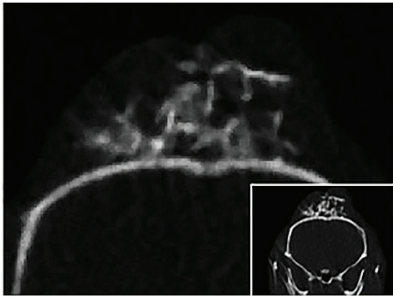

Fig. $2 g$

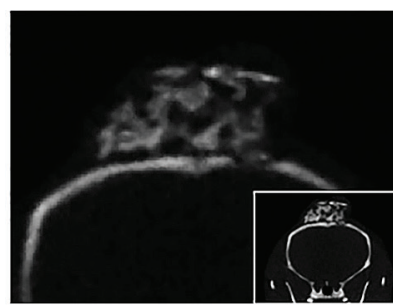

Fig. $2 \mathrm{~h}$

Villanueva bone staining and micro CT scans of the transplanted osteophyte (a to d) and cancellous bone (e to h). Resin-embedded undecalcified specimens were observed under ( $a$ and e) natural and ( $b$ and f) fluorescent light. (b) The green fluorescent line, corresponding to area of mineralisation, is observed along almost the entire circumference of the transplanted osteophyte (arrowheads). (f) In contrast, the green line is limited to only one area for the transplanted cancellous bone (arrowheads). For (b) and ( $f$ ), arrows represent the mineralised surface of the calvarial bone (original magnification, $\times 80$ ). Micro CT scans at ( $c$ and $g$ ) three weeks and ( $d$ and $h$ ) six weeks after transplantation are shown as a reference. Arrowheads in figure $d$ represent the newly formed bone, six weeks after osteophyte transplantation.

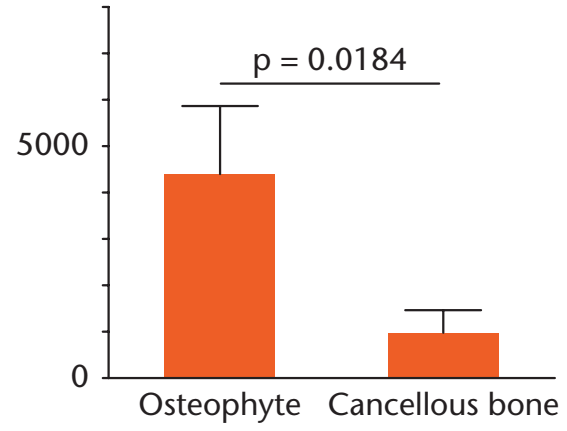

Fig. $3 a$

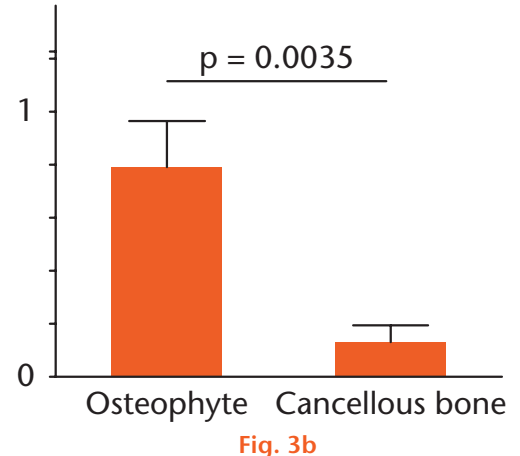

Fig. 3b

Graphs showing quantification of the area of mineralisation (a) and the ratio of labelled area: total area of the graft (b) is shown, with results reported as the mean and standard deviation. Statistical analysis was performed using one-way analysis of variance (both $n=3)$.

rose significantly higher in the osteophyte-conditioned medium than in the cancellous bone-conditioned medium.

Quantitative PCR analysis revealed the effect of each conditioned medium on osteoblast differentiation factor expression. MG-63 cells are known to exhibit a relatively pre-mature osteoblast phenotype. ${ }^{15}$ After a 72-hour incubation of the cells in each medium, the differentiation marker collagen type 1 alpha 1 (COL1A1) was significantly increased in the osteophyte-conditioned medium group, compared with the untreated control and cancellous bone-conditioned medium groups $(p<0.0001$ and $p=0.0003$, respectively; Fig. 6). In order to confirm gene expression of a more advanced stage of differentiation, Saos- 2 cells were similarly cultured in each medium. The expression of alkaline phosphatase (ALP), a marker of a relatively later stage of differentiation, was 

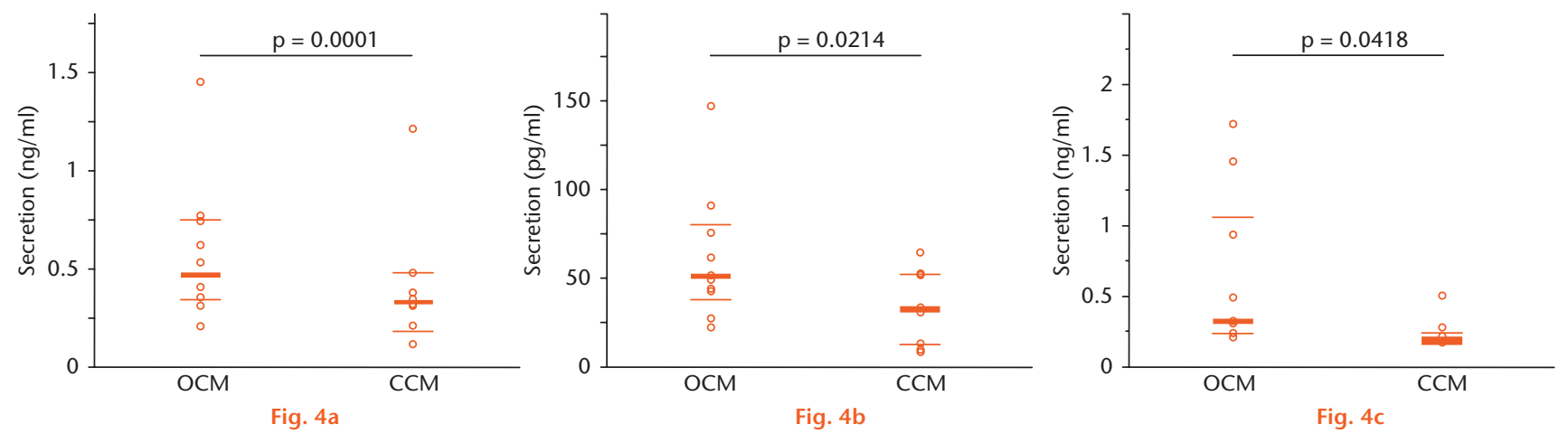

Graphs showing secretion of transforming growth factor (TGF)- $\beta 1$ (a), bone morphogenetic protein (BMP)-2 (b) and insulin-like growth factor (IGF)-1 (c) in each conditioned medium assessed by enzyme-linked immunosorbent assay, with results reported as the median and interquartile range. The values are as follows: TGF- $\beta 1,471$ (344.5 to 750) versus 333 (189.5 to 478.3 ) pg/ml; BMP-2, 47.75 (25.88 to 79.35) versus 32 (12.22 to 52.3) pg/ml; and IGF-1, 314.5 (230.3 to 1060.8 ) versus 191 (170 to 228.5$) \mathrm{pg} / \mathrm{ml}$. Statistical analysis was performed using Wilcoxon rank sum test (all $\mathrm{n}=10$ ). OCM, osteophyte-conditioned medium; CCM, cancellous bone-conditioned medium.

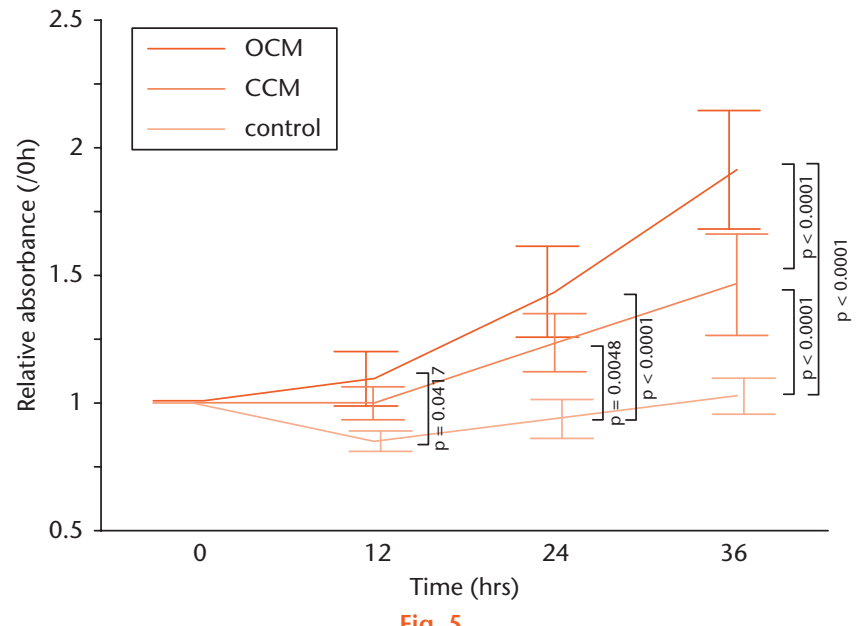

Fig. 5

Graphs showing the effect of each conditioned medium on osteoblast proliferation, assessed using the MTS assay, showing a significantly higher MG-63 cell proliferation rate, up to 36 hours, in the osteophyte medium group than in the untreated control group: 12 hours: 1.09 fold, SD 0.107 versus 0.847 fold, SD 0.0402; 24 hours: 1.43 fold, SD 0.179 versus 0.934 fold, SD 0.0758 and 36 hours: 1.91 fold, SD 0.232 versus 1.02 fold, SD 0.0695 . After 36 hours of incubation, the rate was even higher than for the cancellous bone group: 1.91 fold, SD 0.232 versus 1.46 fold, SD 0.198. Data were corrected for the absorbance at time zero. Results are reported as mean and standard deviation. Statistical analysis was performed using one-way analysis of variance with Tukey's post hoc test (all $\mathrm{n}=10$ ). OCM, osteophyte-conditioned medium; CCM, cancellous bone-conditioned medium.

significantly increased in the osteophyte-conditioned medium group, compared with the untreated control and cancellous bone-conditioned medium groups $(p=0.0026$ and $p=0.0091$, respectively). In contrast, the expression of osteocalcin (BGLAP), a marker of the terminal stage of differentiation, was inhibited in both conditioned mediums.

Transwell chamber assay for the effects of each medium on osteoblast migration revealed a significantly higher migration of MG-63 cells towards the osteophyteconditioned medium than towards the cancellous boneconditioned medium (202 cells, SD 64.7 versus 83.9 cells,
SD 72.6, $p=0.0002$ ) and untreated control (202 cells, SD 64.7 versus 20.2 cells, SD 6.83, $p<0.0001$ ) after six hours of incubation (Fig. 7).

\section{Discussion}

Although the nature of osteophyte formation, as a pathological joint alteration or an adaptation to instability is unknown, the molecular mechanism of osteophyte development is fairly well defined. Mesenchymal stem cells within periosteal or synovial tissue differentiate into chondrocytes and form osteophytes via endochondral ossification, with an additional contribution of intramembranous bone formation within the covering fibrous tissue and bone formation within the marrow spaces. ${ }^{7}$ Expression of growth factors capable of inducing osteogenesis and chondrogenesis, such as TGF- $\beta 1$, BMP- 2 and IGF-1, is upregulated during osteophyte formation. . $-10^{-10}$ Therefore, osteophytes may contain stronger bone-forming potency than cancellous bone as a source of bone graft. We have previously confirmed the clinical feasibility of using osteophytes as autologous bone grafts to fill the gap at the osteotomy site during OW-HTO, with evidence of early bone union. ${ }^{13}$ Here, we corroborated our clinical findings, providing evidence of enhanced bone mineralisation in vivo using a mouse model, and enhanced proliferation, differentiation and chemotaxis of osteoblasts, in vitro using osteoblast-like cell lines, with transplanted osteophytes compared with cancellous bone.

The clinical significance of our findings, however, is limited by the small number of mice used in quantitative in vivo experiments, the use of MG-63 and Saos- 2 cells as osteoblast-like cell lines in vitro, and the lack of authentication of these cell lines. As MG-63 cells exhibit a relatively premature osteoblast phenotype and Saos-2 cells a relatively mature phenotype, we used these two cell types to confirm graft effects on osteoblast differentiation as a function of the stage of differentiation. Although the most desirable method would have been to use primary 

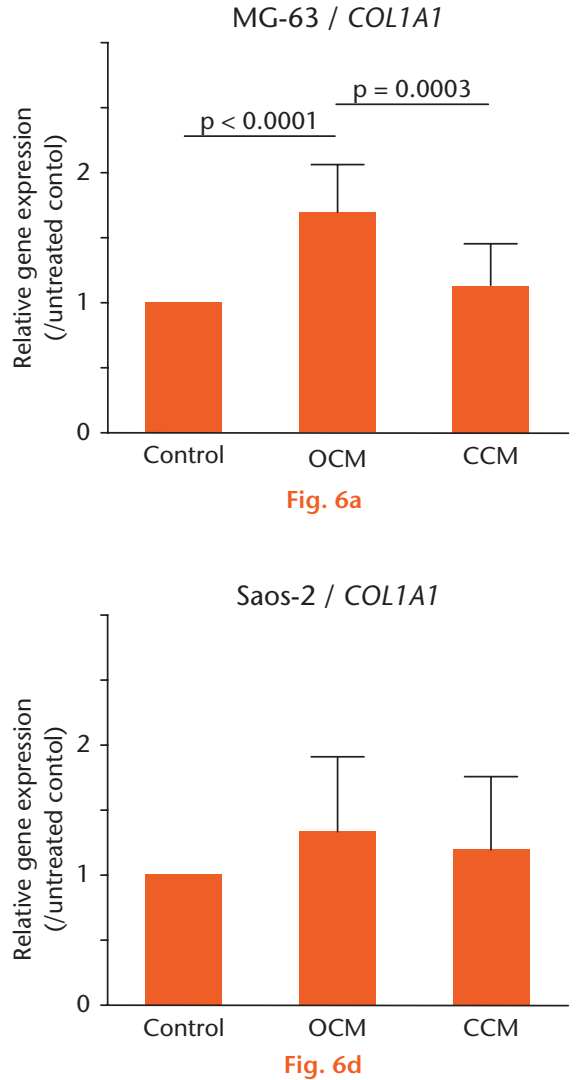

MG-63 / ALP
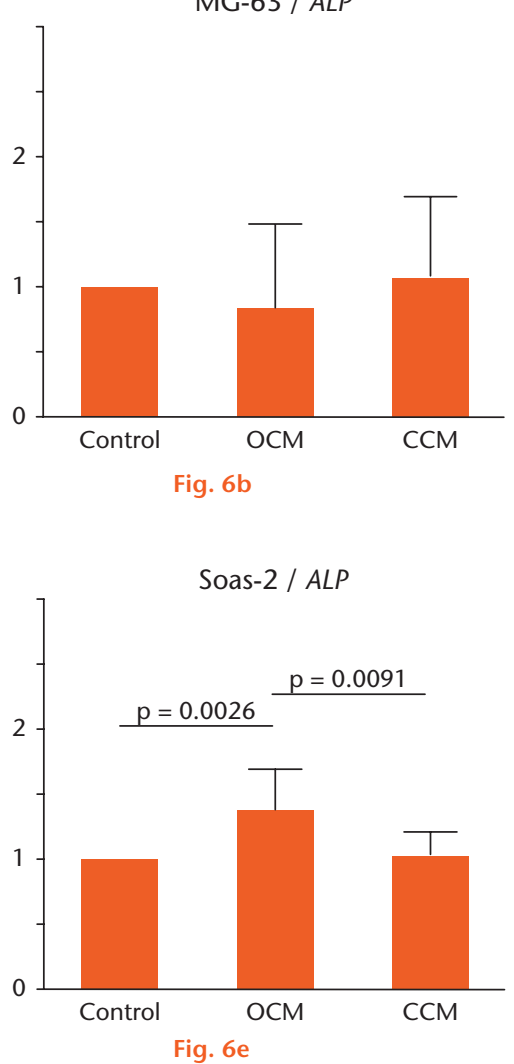

MG-63 / BGLAP

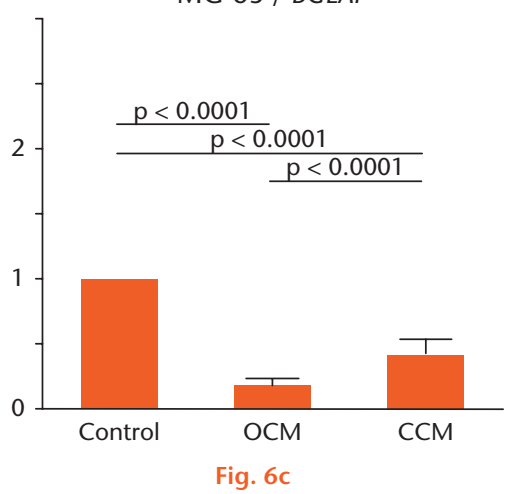

Saos-2 / BGLAP

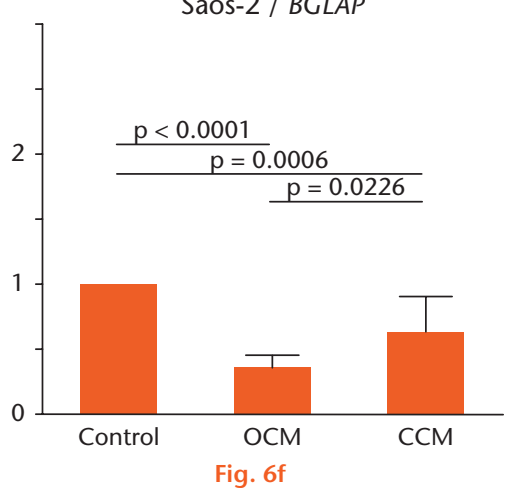

Gene expression is shown after 72 hours of incubation of MG-63 cells or Saos- 2 cells, with the data corrected for expression of the housekeeping gene GAPDH. The value of each mRNA expression relative to that for the untreated control is indicated. The results are reported as mean and standard deviation. Statistical analysis was performed using one-way analysis of variance with Tukey's post hoc test (all $\mathrm{n}=10)$. OCM, osteophyte-conditioned medium; CCM, cancellous bone-conditioned medium.

osteoblasts isolated from humans, the similarities of MG-63 and Saos-2 to human osteoblasts have been established. ${ }^{15}$ Furthermore, although we have presupposed the clinical feasibility of using osteophyte grafts in OW-HTO procedures, there are points that need to be considered. First, the amount of osteophytes obtained during surgery is commonly insufficient to fill the gap at the osteotomy site, particularly in the presence of mild deformity. In our practice, as we consider bone union at the lateral hinge of the osteotomy to be critical, we specifically localised our osteophyte graft in this area, and no additional bone graft was placed. No complications, such as leaving bone fragments inside the knee joint or the prolonged haemarthrosis, have been reported. ${ }^{13}$ Future studies will need to evaluate the feasibility and usefulness of osteophyte grafting to treat bone defects in other situations, including arthroplasty, osteotomy for hip OA or hallux valgus, and fractures around the knee associated with osteoarthritic changes.

The more active mineralisation of bone with transplantation of osteophytes than with cancellous bone likely reflects the differential secretion of growth factors by each graft type. Growth factors, such as TGF- $\beta 1$, BMP-2 and IGF-1, are expressed during osteophyte development, including a strong phosphorylation of Smad2, which has been described in a mouse model. ${ }^{16}$ Moreover, injection of TGF- $\beta 1$ stimulates osteophyte formation similarly to that of osteophytes observed in murine OA models. ${ }^{17}$ Therefore, TGF- $\beta 1$ plays an important role in the initiation of osteophyte formation during OA. BMP-2 is also expressed in hypertrophic chondrocytes of osteophytes, while IGF-1 is expressed in proliferating perichondral cells, chondrocytes and osteoblasts. ${ }^{8}$ In animal models, BMP-2 and IGF-1 treatment stimulated type II collagen and aggrecan expression, ${ }^{18,19}$ indicative of their contribution to chondrogenesis and the development of osteophytes.

With regard to osteogenesis, local application of TGF$\beta 1$, IGF-1 and BMP-2 at fracture or osteotomy sites accelerates bone healing in animal models. ${ }^{20,21}$ In the early process of osteogenesis, proliferation of osteoprogenitor cells, inside and/or around the graft, and recruitment of the osteoblastic lineage are needed, with TGF- $\beta 1$ signalling known to promote this recruitment through the mitogen-activated protein kinases and Smad2/3 pathways. ${ }^{22}$ The importance of BMP-2 and IGF-1 to the differentiation and maturation of the osteoblastic lineage is well recognised, ${ }^{23,24}$ with TGF- $\beta 1$ further inducing osteoblast proliferation. ${ }^{25}$ In our study, we identified a higher expression of these growth factors 

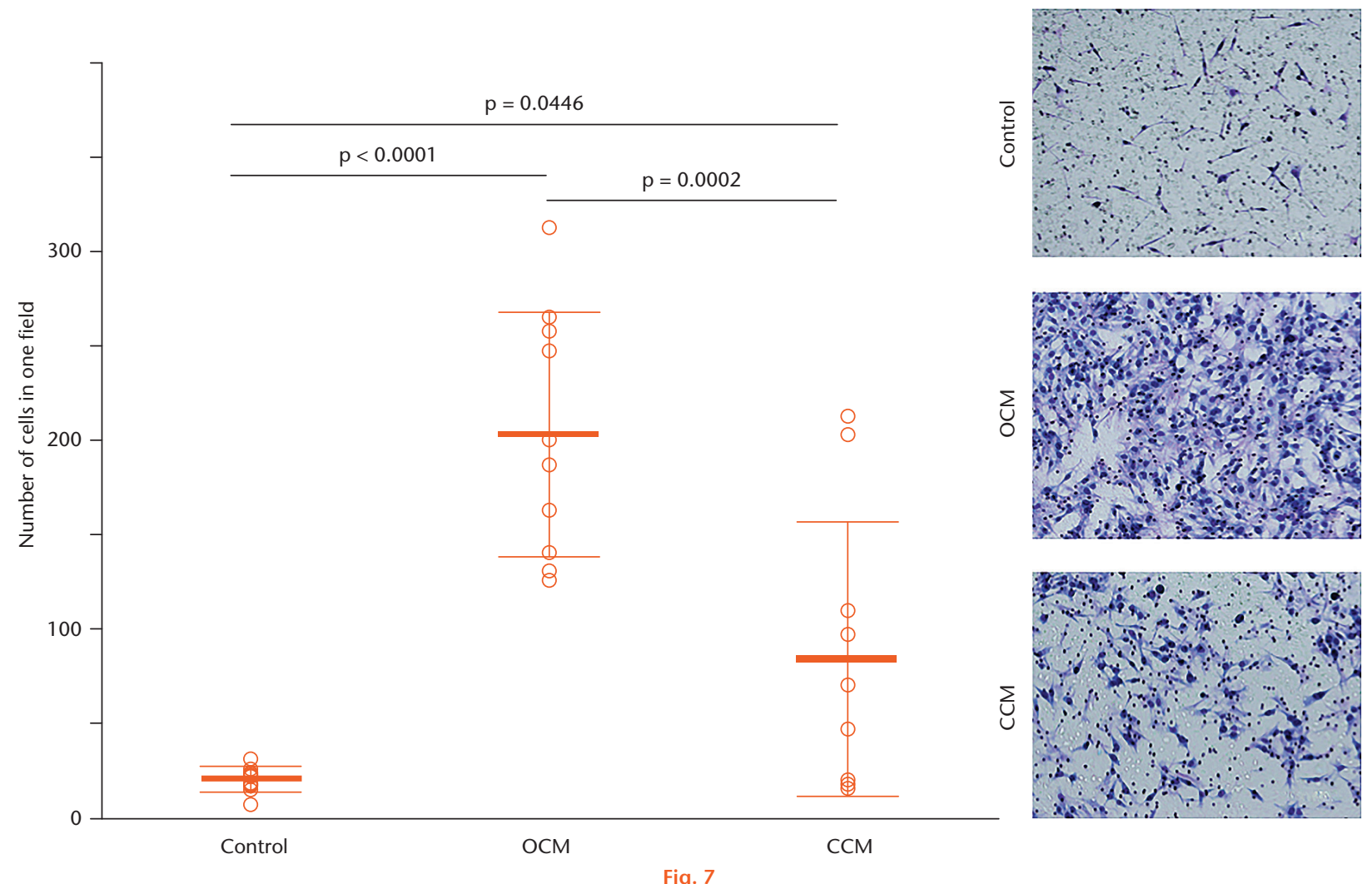

Migration assay using Transwell chambers, showing a significantly higher migration activity of MG-63 cells towards osteophyte-conditioned medium than towards cancellous bone-conditioned medium and the untreated control after six hours of incubation. The cells were counted in three randomly selected microscopic fields $(\times 400)$ and the mean taken, with representative images shown for the ten experiments (original magnification, $\times 400)$. The results are reported as mean and standard deviation. Statistical analysis was performed using one-way analysis of variance with Tukey's post hoc test (all $n=10$ ). OCM, osteophyteconditioned medium; CCM, cancellous bone-conditioned medium.

in the osteophyte-conditioned medium, compared with the cancellous bone-conditioned medium, using ELISA. To our knowledge, we are the first to have reported the amount of growth factors secreted by osteophytes into their surroundings. Growth factors exuded from osteophytes may play a key role in mineralisation around the graft. Although stronger mineralisation was confirmed in the in vivo osteophyte transplantation model, the expression of osteocalcin from osteoblasts, recognised as a terminal differentiation marker, was inhibited when osteoblasts were cultured, in vitro, in the osteophyte-conditioned medium. We do know whether TGF- $\beta 1$ is a potent inhibitor of terminal osteoblast differentiation and mineralisation, ${ }^{26}$ and therefore, this discrepancy between in vivo and in vitro results likely reflects the involvement of various factors other than the three growth factors we measured, or it is likely that TGF- $\beta 1$ activation would be different in each microenvironment.

In order to form new bone in the appropriate location, osteoblasts need to migrate and gather at the edge of the existing bone, with IGF-1 involved in the recruitment of osteoblasts at the site of bone formation. ${ }^{27}$ The osteophyte-conditioned medium, which contained large amounts of IGF-1, induced greater migration of MG-63 cells than cancellous bone. Previous research, using a mouse allograft transplantation model, reported that osteoblasts surrounding bone graft are gradually replaced by host cells over a period of six weeks. ${ }^{28}$ IGF-1 secreted by osteophytes could, therefore, make a great contribution to bone mineralisation by recruiting osteoblasts from its surroundings.

In conclusion, the greater osteoblastic efficacy of osteophytes over cancellous bone is supported by the greater amount of newly mineralised area around the graft in vivo at six weeks following transplantation. Moreover, osteophytes express a number of factors which favour bone union such as TGF- $\beta 1$, BMP- 2 , and IGF- 1 , with the combined effects of these growth factors likely to contribute to osteoblast proliferation, differentiation and migration. Their usefulness as bone grafts should be considered. Further research is required to further evaluate the feasibility of osteophytes for other applications requiring early bone union, such as arthroplasty with bone defects, or other osteotomies of arthritic joints.

\section{Supplementary material}

$\because$ A table showing primer sequences is available online at www.bjr.boneandjoint.org.uk 


\section{References}

1. Sen MK, Miclau T. Autologous iliac crest bone graft: should it still be the gold standard for treating nonunions? Injury 2007;38:S75-S80.

2. Fillingham Y, Jacobs J. Bone grafts and their substitutes. Bone Joint J 2016;98-B(1 Suppl A):6-9.

3. Younger EM, Chapman MW. Morbidity at bone graft donor sites. J Orthop Trauma 1989;3:192-195

4. Gelse K, Söder S, Eger W, et al. Osteophyte development-molecular characterization of differentiation stages. Osteoarthritis Cartilage 2003;11: 141-148.

5. van der Kraan PM, van den Berg WB. Osteophytes: relevance and biology. Osteoarthritis Cartilage 2007;15:237-244.

6. Dodds RA, Gowen M. The growing osteophyte: a model system for the study of human bone development and remodeling in situ. J Histotechnol 1994;17:37-45.

7. Zoricic S, Maric I, Bobinac D, Vukicevic S. Expression of bone morphogenetic proteins and cartilage-derived morphogenetic proteins during osteophyte formation in humans. J Anat 2003;202:269-277.

8. Okazaki K, Jingushi S, Ikenoue T, et al. Expression of insulin-like growth factor I messenger ribonucleic acid in developing osteophytes in murine experimental osteoarthritis and in rats inoculated with growth hormone-secreting tumor. Endocrinology 1999;140:4821-4830.

9. Nakase T, Miyaji T, Tomita T, et al. Localization of bone morphogenetic protein-2 in human osteoarthritic cartilage and osteophyte. Osteoarthritis Cartilage 2003;11: 278-284.

10. Uchino M, Izumi T, Tominaga T, et al. Growth factor expression in the osteophytes of the human femoral head in osteoarthritis. Clin Orthop Relat Res 2000;377:119-125.

11. Hernigou P, Medevielle D, Debeyre J, Goutallier D. Proximal tibial osteotomy for osteoarthritis with varus deformity. A ten to thirteen-year follow-up study. J Bone Joint Surg [Am]1987;69-A:332-354.

12. Lash NJ, Feller JA, Batty LM, et al. Bone grafts and bone substitutes for opening-wedge osteotomies of the knee: a systematic review. Arthroscopy 2015;31:720-730

13. Akiyama T, Okazaki K, Mawatari T, Ikemura S, Nakamura S. Autologous osteophyte grafting for open-wedge high tibial osteotomy. Arthrosc Tech 2016;5: e989-e995

14. Pottenger LA, Phillips FM, Draganich LF. The effect of marginal osteophytes on reduction of varus-valgus instability in osteoarthritic knees. Arthritis Rheum 1990:33:853-858.

15. Pautke C, Schieker M, Tischer T, et al. Characterization of osteosarcoma cell lines MG-63, Saos-2 and U-2 OS in comparison to human osteoblasts. Anticancer Res 2004; $24: 3743-3748$

16. Blaney Davidson EN, Vitters EL, van der Kraan PM, van den Berg WB. Expression of transforming growth factor- $\beta$ (TGFbeta) and the TGFbeta signalling molecule SMAD-2P in spontaneous and instability-induced osteoarthritis: role in cartilage degradation, chondrogenesis and osteophyte formation. Ann Rheum Dis 2006;65:1414-1421.

17. van Beuningen HM, van der Kraan PM, Arntz OJ, van den Berg WB. Transforming growth factor-beta 1 stimulates articular chondrocyte proteoglycan synthesis and induces osteophyte formation in the murine knee joint. Lab Invest 1994;71:279-290.

18. Hanada K, Solchaga LA, Caplan Al, et al. BMP-2 induction and TGF- $\beta$ modulation of rat periosteal cell chondrogenesis. J Cell Biochem 2001;81:284-294.
19. Fukumoto T, Sperling JW, Sanyal A, et al. Combined effects of insulin-like growth factor- 1 and transforming growth factor- $\beta 1$ on periosteal mesenchymal cells during chondrogenesis in vitro. Osteoarthritis Cartilage 2003;11:55-64

20. Schmidmaier G, Wildemann B, Bail H, et al. Local application of growth factors (insulin-like growth factor-1 and transforming growth factor-beta1) from a biodegradable poly(D,L-lactide) coating of osteosynthetic implants accelerates fracture healing in rats. Bone 2001;28:341-350.

21. Raschke $\mathbf{M}$, Wildemann $\mathbf{B}$, Inden $\mathbf{P}$, et al. Insulin-like growth factor-1 and transforming growth factor- $\beta 1$ accelerates osteotomy healing using polylactidecoated implants as a delivery system: a biomechanical and histological study in minipigs. Bone 2002;30:144-151.

22. Matsunobu $\mathbf{T}$, Torigoe $\mathbf{K}$, Ishikawa $\mathbf{M}$, et al. Critical roles of the TGF- $\beta$ type receptor ALK5 in perichondrial formation and function, cartilage integrity, and osteoblast differentiation during growth plate development. Dev Bio/ 2009;332:325-338.

23. Chen D, Zhao M, Mundy GR. Bone morphogenetic proteins. Growth Factors 2004;22:233-241.

24. Xian L, Wu X, Pang L, et al. Matrix IGF-1 maintains bone mass by activation of mTOR in mesenchymal stem cells. Nat Med 2012:18:1095-1101.

25. Bosetti M, Boccafoschi F, Leigheb M, Cannas MF. Effect of different growth factors on human osteoblasts activities: a possible application in bone regeneration for tissue engineering. Biomol Eng 2007;24:613-618

26. Spinella-Jaegle S, Roman-Roman S, Faucheu C, et al. Opposite effects of bone morphogenetic protein-2 and transforming growth factor-beta1 on osteoblast differentiation. Bone 2001:29:323-330.

27. Nakasaki M, Yoshioka K, Miyamoto Y, et al. IGF-I secreted by osteoblasts acts as a potent chemotactic factor for osteoblasts. Bone 2008;43:869-879.

28. Yano K, Yasuda $\mathbf{H}$, Takaoka K, et al. Fate, origin and roles of cells within free bone grafts. J Orthop Sci2015;20:390-396.

Funding Statement

We thank Ms Akemi Ito at the Ito Bone Histomorphometry Institute (Niigata, Japan) for her histomorphological support and Ms H. Kimura for her assistance in histological preparations. This study was partially supported by JSPS KAKENHI Grant Number 15K10478.

K. Okazaki, Y. Akasaki and Y. Nakashima declare that they have received a grant from the Japan Society for the Promotion of Science unrelated to this work. K. Okazaki this work.

Author Contribution

- K. Ishihara: Conceived and designed the experiments, analysed the data, wrote and revised the paper, read and approved the final submitted manuscript, performed the experiments

- K. Okazaki: Conceived and designed the experiments, analysed the data, wrote and revised the paper, read and approved the final submitted manuscript, performed the experiments

T. Akiyama: Conceived and designed the experiments, analysed the data, wrote and revised the paper, read and approved the final submitted manuscript

- Y. Akasaki: Conceived and designed the experiments, analysed the data, wrote and revised the paper, read and approved the final submitted manuscript

Y. Nakashima: Conceived and designed the experiments, analysed the data, wrote and revised the paper, read and approved the final submitted manuscript

ICMJE Conflicts of Intrest

- None declared

(c) 2017 Okazaki et al. This is an open-access article distributed under the terms of the Creative Commons Attributions licence (CC-BY-NC), which permits unrestricted use, distribution, and reproduction in any medium, but not for commercial gain, provided the original author and source are credited. 